\title{
Morphing Planar Graph Drawings Efficiently ${ }^{\star}$
}

\author{
Patrizio Angelini ${ }^{1}$, Fabrizio Frati ${ }^{2}$, Maurizio Patrignani ${ }^{1}$, and Vincenzo Roselli ${ }^{1}$ \\ 1 Engineering Department, Roma Tre University, Italy \\ \{angelini, patrignani, roselli\}edia.uniroma3.it \\ 2 School of Information Technologies, The University of Sydney, Australia \\ brillo@it.usyd.edu.au
}

\begin{abstract}
A morph between two straight-line planar drawings of the same graph is a continuous transformation from the first to the second drawing such that planarity is preserved at all times. Each step of the morph moves each vertex at constant speed along a straight line. Although the existence of a morph between any two drawings was established several decades ago, only recently it has been proved that a polynomial number of steps suffices to morph any two planar straight-line drawings. Namely, at SODA 2013, Alamdari et al. [1] proved that any two planar straight-line drawings of a planar graph can be morphed in $O\left(n^{4}\right)$ steps, while $O\left(n^{2}\right)$ steps suffice if we restrict to maximal planar graphs.

In this paper, we improve upon such results, by showing an algorithm to morph any two planar straight-line drawings of a planar graph in $O\left(n^{2}\right)$ steps; further, we show that a morph with $O(n)$ steps exists between any two planar straight-line drawings of a series-parallel graph.
\end{abstract}

\section{Introduction}

A planar morph between two planar drawings of the same plane graph is a continuous transformation from the first drawing to the second one such that planarity is preserved at all times. The problem of deciding whether a planar morph exists for any two drawings of any graph dates back to 1944, when Cairns [7] proved that any two straight-line drawings of a maximal planar graph can be morphed one into the other while maintaining planarity. In 1981, Grünbaum and Shephard [10] introduced the concept of linear morph, that is a continuous transformation in which each vertex moves at uniform speed along a straight-line trajectory. With this further requirement, however, planarity cannot always be maintained for any pair of drawings. Hence, the problem has been subsequently studied in terms of the existence of a sequence of linear morphs, also called morphing steps, transforming a drawing into another while maintaining planarity. The first result in this direction is the one of Thomassen [13], who proved that a sequence of morphing steps always exists between any two straight-line drawings of the same plane graph. Further, if the two input drawings are convex, this property is maintained throughout the morph, as well. However, the number of morphing steps used by the algorithm of Thomassen might be exponential in the number of vertices.

\footnotetext{
* Part of the research was conducted in the framework of ESF project 10-EuroGIGA-OP-003

GraDR "Graph Drawings and Representations".
} 
Recently, the problem of computing planar morphs gained increasing research attention. The case in which edges are not required to be straight-line segments has been addressed in [11], while morphs between orthogonal graph drawings preserving planarity and orthogonality have been explored in [12]. Morphs preserving more general edge directions have been considered in [6]. Also, the problem of "topological morphing", in which the planar embedding is allowed to change, has been addressed in [2].

In a paper appeared at SODA 2013, Alamdari et al. [1] tackled again the original setting in which edges are straight-line segments and linear morphing steps are required. Alamdari et al. presented the first morphing algorithms with a polynomial number of steps in this setting. Namely, they presented an algorithm to morph straight-line planar drawings of maximal plane graphs with $O\left(n^{2}\right)$ steps and of general plane graphs with $O\left(n^{4}\right)$ steps, where $n$ is the number of vertices of the graph.

In this paper we improve upon the result of Alamdari et al. [1], providing a more efficient algorithm to morph general plane graphs. Namely, our algorithms uses $O\left(n^{2}\right)$ linear morphing steps. Further, we provide a morphing algorithm with a linear number of steps for a non-trivial class of planar graphs, namely series-parallel graphs. These two main results are summarized in the following theorems.

Theorem 1. Let $\Gamma_{a}$ and $\Gamma_{b}$ be two drawings of the same plane series-parallel graph $G$. There exists a morph $\left\langle\Gamma_{a}, \ldots, \Gamma_{b}\right\rangle$ with $O(n)$ steps transforming $\Gamma_{a}$ into $\Gamma_{b}$.

Theorem 2. Let $\Gamma_{s}$ and $\Gamma_{t}$ be two drawings of the same plane graph $G$. There exists a morph $\left\langle\Gamma_{s}, \ldots, \Gamma_{t}\right\rangle$ with $O\left(n^{2}\right)$ steps transforming $\Gamma_{s}$ into $\Gamma_{t}$.

The rest of the paper is organized as follows. Section 2 contains preliminaries and basic terminology. Section 3 describes an algorithm to morph series-parallel graphs. Section 4 describes an algorithm to morph plane graphs. Section 5 provides geometric details for the morphs described in Sections 3 and 4 Finally, Section 6 contains conclusions and open problems. Because of space limitations, some proofs are omitted or sketched. Full proofs can be found in the extended version of the paper [4].

\section{Preliminaries}

A straight-line planar drawing $\Gamma$ (in the following simply drawing) of a graph $G(V, E)$ maps vertices in $V$ to distinct points of the plane and edges in $E$ to non-intersecting open straight-line segments between their end-vertices. Given a vertex $v$ of a graph $G$, we denote by $\operatorname{deg}(v)$ the degree of $v$ in $G$, that is, the number of vertices adjacent to $v$. A planar drawing $\Gamma$ partitions the plane into connected regions called faces. The unbounded face is the external face. Also, $\Gamma$ determines a clockwise order of the edges incident to each vertex. Two planar drawings are equivalent if they determine the same clockwise ordering of the incident edges around each vertex and if they have the same external face. A planar embedding is an equivalence class of planar drawings. A plane graph is a planar graph with a given planar embedding.

A series-parallel graph $G$ is a planar graph that does not contain the complete graph on four vertices as a minor. A plane series-parallel graph is a graph together with a planar embedding. Let $G$ be a plane biconnected series-parallel graph and let $e$ be an edge 
incident to its outer face. Graph $G$ has a unique decomposition tree $T_{e}$ rooted at $e$ having nodes of three types: Q-, S-, and P-nodes. A Q-node represents a single edge, while an S-node (a P-node) $\mu$ represents a series (a parallel, respectively) composition of the sugraphs associated to the subtrees of $T_{e}$ rooted at the children of $\mu$. An embedding of $G$ naturally induces an ordering for the children of each node of $T_{e}$.

A (linear) morphing step $\left\langle\Gamma_{1}, \Gamma_{2}\right\rangle$, also referred to as linear morph, of two straightline planar drawings $\Gamma_{1}$ and $\Gamma_{2}$ of a plane graph $G$ is a continuous transformation of $\Gamma_{1}$ into $\Gamma_{2}$ such that all the vertices simultaneously start moving from their positions in $\Gamma_{1}$ and, moving along a straight-line trajectory, simultaneously stop at their positions in $\Gamma_{2}$ so that no crossing occurs between any two edges during the transformation. A morph $\left\langle\Gamma_{s}, \ldots, \Gamma_{t}\right\rangle$ of two straight-line planar drawings $\Gamma_{s}$ into $\Gamma_{t}$ of a plane graph $G$ is a finite sequence of morphing steps that transforms $\Gamma_{s}$ into $\Gamma_{t}$. Let $u$ and $w$ be two vertices of $G$ such that edge $(u, w)$ belongs to $G$ and let $\Gamma$ be a straight-line planar drawing of $G$. The contraction of $u$ onto $w$ results in $(i)$ a graph $G^{\prime}=G /(u, w)$ not containing $u$ and such that each edge $(u, x)$ of $G$ is replaced by an edge $(w, x)$ in $G^{\prime}$, and $(i i)$ a straight-line drawing $\Gamma^{\prime}$ of $G^{\prime}$ such that each vertex different from $v$ is mapped to the same point as in $\Gamma$. In the following, the contraction of an edge $(u, w)$ will be only applied if the obtained drawing $\Gamma^{\prime}$ is planar. The uncontraction of $u$ from $w$ in $\Gamma^{\prime}$ yields a straight-line planar drawing $\Gamma^{\prime \prime}$ of $G$. A morph in which contractions are performed, possibly together with other morphing steps, is a pseudo-morph. Let $v$ be a vertex of $G$ and let $G^{\prime}$ be the graph obtained by removing $v$ and its incident edges from $G$. Let $\Gamma^{\prime}$ be a planar straight-line drawing of $G^{\prime}$. The kernel of $v$ in $\Gamma^{\prime}$ is the set $P$ of points such that straight-line segments can be drawn in $\Gamma^{\prime}$ connecting each point $p \in P$ to each neighbor of $v$ in $G$ without intersecting any edge in $\Gamma^{\prime}$.

\section{Morphing Series-Parallel Graph Drawings in $\mathrm{O}(\mathrm{n})$ Steps}

In this section we show an algorithm to compute a pseudo-morph between any two drawings of the same plane series-parallel graph $G$. In Section 3.1 we assume that $G$ is biconnected, and in Section 3.2 we show how to remove this assumption, thus proving the following theorem.

Theorem 3. Let $\Gamma_{a}$ and $\Gamma_{b}$ be two drawings of the same plane series-parallel graph $G$. There exists a pseudo-morph $\left\langle\Gamma_{a}, \ldots, \Gamma_{b}\right\rangle$ with $O(n)$ steps transforming $\Gamma_{a}$ into $\Gamma_{b}$.

\subsection{Biconnected Series-Parallel Graphs}

Our approach consists of morphing any drawing $\Gamma$ of a biconnected plane series-parallel graph $G$ into a "canonical drawing" $\Gamma^{*}$ of $G$ in a linear number of steps. As a consequence, any two drawings $\Gamma_{1}$ and $\Gamma_{2}$ of $G$ can be transformed one into the other in a linear number of steps, by morphing $\Gamma_{1}$ to $\Gamma^{*}$ and $\Gamma^{*}$ to $\Gamma_{2}$.

A canonical drawing $\Gamma^{*}$ of a biconnected plane series-parallel graph $G$ is defined as follows. The decomposition tree $T_{e}$ of $G$ is traversed top-down and a suitable geometric region of the plane is assigned to each node $\mu$ of $T_{e}$; such a region will contain the drawing of the series-parallel graph associated with $\mu$. The regions assigned to the nodes 


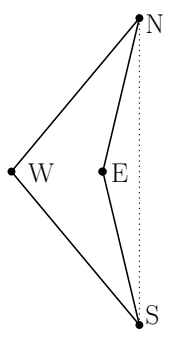

(a)

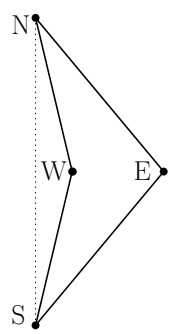

(b)

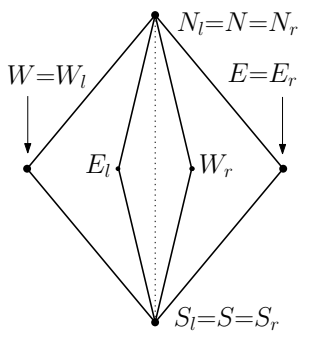

(c)

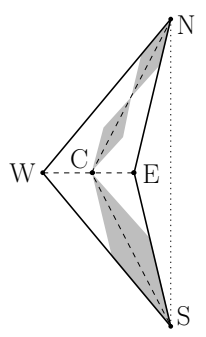

(d)

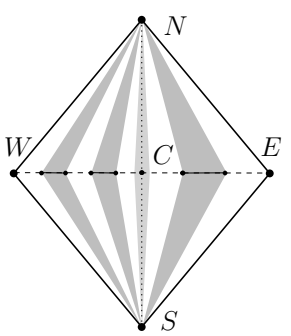

(e)

Fig. 1. (a) A left boomerang. (b) A right boomerang. (c) A diamond. (d) Diamonds inside a boomerang. (e) Boomerangs (and a diamond) inside a diamond.

of $T_{e}$ are similar to those used in [5]3] to construct monotone drawings. Namely, we define three types of regions: Left boomerangs, right boomerangs, and diamonds. A left boomerang is a quadrilateral with vertices $N, E, S$, and $W$ such that $E$ is inside triangle $\triangle(N, S, W)$, where $|\overline{N E}|=|\overline{S E}|$ and $|\overline{N W}|=|\overline{S W}|$ (see Fig. 11(a)). A right boomerang is defined symmetrically, with $E$ playing the role of $W$, and vice versa (see Fig. 1(b)]. A diamond is a convex quadrilateral with vertices $N, E, S$, and $W$, where $|\overline{N W}|=|\overline{N E}|=|\overline{S W}|=|\overline{S E}|$. Observe that a diamond contains a left boomerang $N_{l}, E_{l}, S_{l}, W_{l}$ and a right boomerang $N_{r}, E_{r}, S_{r}, W_{r}$, where $S=S_{l}=S_{r}$, $N=N_{l}=N_{r}, W=W_{l}$, and $E=E_{r}$ (see Fig. 1(c)).

We assign boomerangs (either left or right, depending on the embedding of $G$ ) to S-nodes and diamonds to P- and Q-nodes, as follows.

First, consider the Q-node $\rho$ corresponding to the root edge $e$ of $G$. Draw edge $e$ as a segment between points $(0,1)$ and $(0,-1)$. Also, if $\rho$ is adjacent to an S-node $\mu$, then assign to $\mu$ the left boomerang $N=(0,1), E=(-1,0), S=(0,-1), W=(-2,0)$ or the right boomerang $N=(0,1), E=(2,0), S=(0,-1), W=(1,0)$, depending on the embedding of $G$; if $\rho$ is adjacent to a P-node $\mu$, then associate to $\mu$ the diamond $N=(0,1), E=(+2,0), S=(0,-1), W=(-2,0)$.

Then, consider each node $\mu$ of $T_{e}(G)$ according to a top-down traversal.

If $\mu$ is an S-node (see Fig. 1(d)], let $N, E, S, W$ be the boomerang associated with it and let $\alpha$ be the angle $\widehat{W N E}$. We associate diamonds to the children $\mu_{1}, \mu_{2}, \ldots, \mu_{k}$ of $\mu$ as follows. Consider the midpoint $C$ of segment $\overline{W E}$. Subdivide $\overline{N C}$ into $\left\lceil\frac{k}{2}\right\rceil$ segments with the same length and $\overline{C S}$ into $\left\lfloor\frac{k}{2}\right\rfloor$ segments with the same length. Enclose each of such segments $\overline{N_{i} S_{i}}$, for $i=1, \ldots, k$, into a diamond $N_{i}, E_{i}, S_{i}, W_{i}$, with ${\widehat{W_{i} N_{i} E_{i}}}_{i}=\alpha$, and associate it with child $\mu_{i}$ of $\mu$.

If $\mu$ is a P-node (see Fig. 1(e), let $N, E, S, W$ be the diamond associated with it. Associate boomerangs and diamonds to the children $\mu_{1}, \mu_{2}, \ldots, \mu_{k}$ of $\mu$ as follows. If a child $\mu_{l}$ of $\mu$ is a Q-node, then left boomerangs are associated to $\mu_{1}, \ldots, \mu_{l-1}$, right boomerangs are associated to $\mu_{l+1}, \ldots, \mu_{k}$, and a diamond is associated to $\mu_{l}$. Otherwise, right boomerangs are associated to all of $\mu_{1}, \mu_{2}, \ldots, \mu_{k}$. We assume that a child $\mu_{l}$ of $\mu$ that is a Q-node exists, the description for the case in which no child of $\mu$ is a Q-node being similar and simpler. We describe how to associate left boomerangs to the 
children $\mu_{1}, \mu_{2}, \ldots, \mu_{l-1}$ of $\mu$. Consider the midpoint $C$ of segment $\overline{W E}$ and consider $2 l$ equidistant points $W=p_{1}, \ldots, p_{2 l}=C$ on segment $\overline{W C}$. Associate each child $\mu_{i}$, with $i=1, \ldots, l-1$, to the quadrilateral $N_{i}=N, E_{i}=p_{2 i}, S_{i}=S, W_{i}=p_{2 i+1}$. Right boomerangs are associated to $\mu_{l+1}, \mu_{l+2}, \ldots, \mu_{k}$ in a symmetric way. Finally, associate $\mu_{l}$ to any diamond such that $N_{l}=N, S_{l}=S, W_{l}$ is any point between $C$ and $E_{l-1}$, and $E_{l}$ is any point between $C$ and $W_{l+1}$.

If $\mu$ is a Q-node, let $N, E, S, W$ be the diamond associated with it. Draw the edge corresponding to $\mu$ as a straight-line segment between $N$ and $S$.

Observe that the above described algorithm constructs a drawing of $G$, that we call the canonical drawing of $G$. We now argue that no two edges $e_{1}$ and $e_{2}$ intersect in the canonical drawing of $G$. Consider the lowest common ancestor $\nu$ of the Q-nodes $\tau_{1}$ and $\tau_{2}$ of $T_{e}$ representing $e_{1}$ and $e_{2}$, respectively. Also, consider the children $\nu_{1}$ and $\nu_{2}$ of $\nu$ such that the subtree of $T_{e}$ rooted at $\nu_{i}$ contains $\tau_{i}$, for $i=1,2$. Such children are associated with internally-disjoint regions of the plane. Since the subgraphs $G_{1}$ and $G_{2}$ of $G$ corresponding to $\nu_{1}$ and $\nu_{2}$, respectively, are entirely drawn inside such regions, it follows that $e_{1}$ and $e_{2}$ do not intersect except, possibly, at common endpoints.

In order to construct a pseudo-morph of a straight-line planar drawing $\Gamma(G)$ of $G$ into its canonical drawing $\Gamma^{*}(G)$, we do the following: (i) We perform a contraction of a vertex $v$ of $G$ into a neighbor of $v$, hence obtaining a drawing $\Gamma\left(G^{\prime}\right)$ of a graph $G^{\prime}$ with $n-1$ vertices; (ii) we inductively construct a pseudo-morph from $\Gamma\left(G^{\prime}\right)$ to the canonical drawing $\Gamma^{*}\left(G^{\prime}\right)$ of $G^{\prime}$; and (iii) we uncontract $v$ and perform a sequence of morphing steps to transform $\Gamma^{*}\left(G^{\prime}\right)$ into the canonical drawing $\Gamma^{*}(G)$ of $G$.

We describe the three steps in more detail.

Let $T_{e}(G)$ be the decomposition tree of $G$ rooted at some edge $e$ incident to the outer face of $G$. Consider a P-node $\nu$ such that the subtree of $T_{e}(G)$ rooted at $\nu$ does not contain any other P-node. This implies that all the children of $\nu$, with the exception of at most one Q-node, are S-nodes whose children are Q-nodes. Hence, the seriesparallel graph $G(\nu)$ associated to $\nu$ is composed of a set of paths connecting its poles $s$ and $t$. Let $p_{1}$ and $p_{2}$ be two paths joining $s$ and $t$ and such that their union is a cycle $\mathcal{C}$ not containing other vertices in its interior (see Fig. 2(a)p. Such paths exist given that the "rest of the graph" with respect to $\nu$ is in the outer face of $G(\nu)$, since the root $e$ of $T_{e}(G)$ is incident to the outer face of $G$. Internally triangulate $\mathcal{C}$ by adding dummy edges (dashed edges of Fig. 2). Cycle $\mathcal{C}$ and the added dummy edges yield a drawing of a biconnected outerplane graph $O$ which, hence, has at least two vertices of degree two.

If there exists a vertex $v$ with $\operatorname{deg}(v)=2$ and $v \neq s, t$ (Case 1), then apply the following contraction. Assume that $v$ belongs to $p_{2}$. Since $O$ is internally triangulated, both the neighbors $v_{1}$ and $v_{2}$ of $v$ belong to $p_{2}$, and they are joined by a dummy edge. We obtain $\Gamma\left(G^{\prime}\right)$ from $\Gamma(G)$ by contracting $v$ onto one of its neighbors, while preserving planarity (see Figs. 2(a) and 2(b)). If $p_{2}$ contains more than two edges (Case 1.1), then $p_{2}$ is replaced in $G^{\prime}$ with a path $p_{2}^{\prime}$ that contains edge $\left(v_{1}, v_{2}\right)$ and does not contain vertex $v$. Otherwise, $p_{2}$ contains exactly two edges $\left(v, v_{1}\right)$ and $\left(v, v_{2}\right)$. If there exists edge $\left(v_{1}, v_{2}\right)$ in $G$ (Case 1.2), then $G^{\prime}=G \backslash\{v\}$. Finally, if edge $\left(v_{1}, v_{2}\right)$ does not exist in $G$ (Case 1.3), then $p_{2}$ is replaced in $G^{\prime}$ with edge $\left(v_{1}, v_{2}\right)$. Otherwise, the only two vertices of degree 2 in $O$ are $s$ and $t$ (Case 2). In this case, one of the two vertices $u_{1}$ and $u_{2}$ of $O$ adjacent to $s$ has degree 3, say $u_{2}$ (since removing $s$ and its incident 


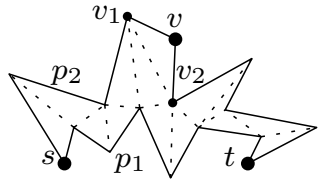

(a)

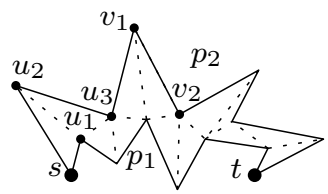

(b)

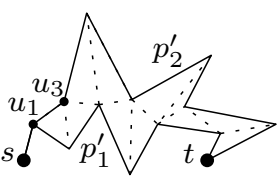

(c)

Fig. 2. The internally triangulated cycle $\mathcal{C}$ formed by paths $p_{1}$ and $p_{2}$. Dummy edges are drawn as dashed lines. (a-b) Vertex $v$ of degree 2 can be contracted onto $v_{1}$. (b-c) Vertex $u_{2}$ of degree 3 can be contracted onto $u_{1}$.

edges from $O$ yields another biconnected outerplane graph with two vertices of degree 2 , namely $t$ and one of $u_{1}$ and $u_{2}$ ). We obtain $\Gamma\left(G^{\prime}\right)$ from $\Gamma(G)$ by contracting $u_{2}$ onto $u_{1}$. Let $u_{3}$ be the neighbor of $u_{1}$ and $u_{2}$ different from $s$. Since the edges incident to $u_{2}$ are contained into triangles $\triangle_{s, u_{1}, u_{2}}$ and $\triangle_{u_{1}, u_{2}, u_{3}}$ during the contraction, planarity is preserved (see Figs. 2(b) and 2(c)). Let $p_{2}^{\prime}$ be the path composed of edge $\left(u_{1}, u_{3}\right)$ and of the subpath of $p_{2}$ between $u_{3}$ and $t$, and let $p_{1}^{\prime}$ be the subpath of $p_{1}$ between $u_{1}$ and $t$. Note that $G^{\prime}$ contains edge $\left(u_{1}, u_{3}\right)$ and does not contain vertex $u_{2}$. In both Case 1 and Case 2, the decomposition tree $T_{e}\left(G^{\prime}\right)$ of $G^{\prime}$ differs from the decomposition tree $T_{e}(G)$ of $G$ only "locally" to $\nu$. A precise description of the differences between $T_{e}(G)$ and $T_{e}\left(G^{\prime}\right)$ can be found in the extended version of the paper [4].

Let $\Gamma\left(G^{\prime}\right)$ be the drawing of the graph $G^{\prime}=G \backslash\{v\}$ obtained after the contraction performed in Case 1 or Case 2. Inductively construct a pseudo-morphing from $\Gamma\left(G^{\prime}\right)$ to the canonical drawing $\Gamma^{*}\left(G^{\prime}\right)$ of $G^{\prime}$ in $c \cdot(n-1)$ steps, where $c$ is a constant. Drawing $\Gamma^{*}(G)$ can be obtained from $\Gamma^{*}\left(G^{\prime}\right)$ by uncontracting $v$ and by performing a constant number of morphing steps, as described in the following.

Here we only describe how to obtain $\Gamma^{*}(G)$ from $\Gamma^{*}\left(G^{\prime}\right)$ if Case 1.1 was applied to contract $v$ into one of its neighbors in $p_{2}$. The other cases can be handled in a similar way (a full description can be found in the extended version of the paper [4]).

Drawings $\Gamma^{*}\left(G^{\prime}\right)$ and $\Gamma^{*}(G)$ coincide except for the fact that path $p_{2}$ in $\Gamma^{*}(G)$ contains $v$, while path $p_{2}^{\prime}$ in $\Gamma^{*}\left(G^{\prime}\right)$ does not contain $v$. Paths $p_{2}^{\prime}$ and $p_{2}$ are drawn inside two equal boomerangs in $\Gamma^{*}\left(G^{\prime}\right)$ and in $\Gamma^{*}(G)$, respectively, however $v$ and some of the vertices of $p_{2}^{\prime}$ need to be moved in order to obtain the drawing of $p_{2}$ as in $\Gamma^{*}\left(G^{\prime}\right)$. Namely, the drawing $\Gamma^{*}\left(p_{2}^{\prime}\right)$ of $p_{2}^{\prime}$ inside the boomerang $N, E, S, W$ associated to $\tau_{2}$ in $\Gamma^{*}\left(G^{\prime}\right)$ is composed of edges lying on two straight-line segments $\overline{N C}$ and $\overline{S C}$, where $C$ is the midpoint of segment $\overline{E W}$ (see Fig. 3(a). The drawing $\Gamma^{*}\left(p_{2}\right)$ of $p_{2}$ in $\Gamma^{*}(G)$ also lies inside $N, E, S, W$ and is composed of edges lying on $\overline{N C}$ and $\overline{S C}$, but vertices lie on different points (see Fig. 3(e)).

With one morphing step, uncontract $v$ from the vertex it had been contracted onto and place it on any point of segment $\overline{v_{1} v_{2}}$ (note that edge $\left(v_{1}, v_{2}\right)$ exists in $G^{\prime}$ and not in $G$; see Fig. 3(b) . Then, in order to redistribute the vertices of $p_{2}$ on $\overline{N C}$ and $\overline{S C}$, perform the following operation. Assume w.l.o.g. that $s$ is on point $N$ and $t$ is on point $S$ in $\Gamma^{*}\left(G^{\prime}\right)$ and in $\Gamma^{*}(G)$. Consider the vertices $w \in p_{2}$ and $w^{\prime} \in p_{2}^{\prime}$ that are placed on point $C$ in $\Gamma^{*}(G)$ and $\Gamma^{*}\left(G^{\prime}\right)$, respectively. Note that either $w=w^{\prime}$ or $\left(w, w^{\prime}\right) \in p_{2}$. If $w=w^{\prime}$, either the subpath $p_{2}(s, w)$ of $p_{2}$ between $s$ and $w$ or the subpath $p_{2}(w, t)$ of $p_{2}$ between $w$ and $t$ has the same drawing in $\Gamma^{*}(G)$ and $\Gamma^{*}\left(G^{\prime}\right)$, say $p_{2}(w, t)$ has 


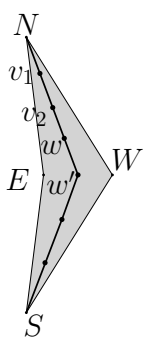

(a)

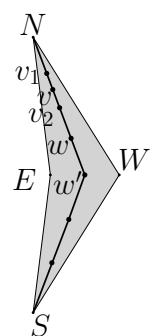

(b)

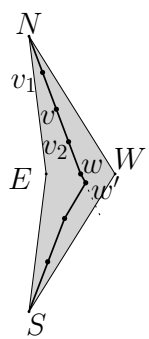

(c)

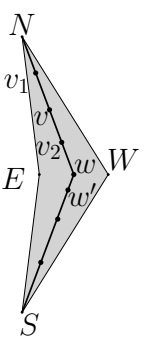

(d)

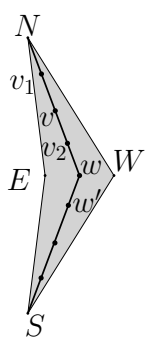

(e)

Fig. 3. Construction of $\Gamma^{*}(G)$ from $\Gamma^{*}\left(G^{\prime}\right)$ when Case 1.1 applied. (a) $\Gamma^{*}\left(p_{2}^{\prime}\right)$. The boomerang associated to $\tau_{2}$ is light-grey. (b) Vertex $v$ is uncontracted and placed on segment $\overline{v_{1} v_{2}}$. (c) Vertices on the path between $s$ and $w$ are placed in their final position, and vertex $w^{\prime}$ is placed arbitrarily close to $C$ on the elongation of $\overline{N C}$. (d) Vertex $w^{\prime}$ is placed on $\overline{C S}$. (e) Vertices on the path between $w$ and $t$ are placed in their final position, hence obtaining $\Gamma^{*}(G)$.

such a property. With one morphing step move the vertices of $p_{2}(s, w)$ on segment $\overline{N C}$ till reaching their positions in $\Gamma^{*}(G)$. If $w \neq w^{\prime}$, assume without loss of generality that $w \in p_{2}\left(s, w^{\prime}\right)$. With one morphing step, move the vertices of $p_{2}(s, w)$ and vertex $w^{\prime}$ along the line through $N$ and $C$, so that the vertices of $p_{2}(s, w)$ reach their positions in $\Gamma^{*}(G)$ and $w^{\prime}$ is placed arbitrarily close to $C$ on the elongation of $\overline{N C}$ (see Fig. 3(c)). With a second morphing step, move $w^{\prime}$ to any point of $\overline{S C}$ between $w$ and its other neighbor in $p_{2}$ (see Fig. 3(d)]. Finally, with a third morphing step, move the vertices of $p_{2}(w, t)$ on segment $\overline{S C}$ till reaching their positions in $\Gamma^{*}(G)$ (see Fig. 3(e)).

\subsection{Simply-Connected Series-Parallel Graphs}

In this section we show how, by preprocessing the input drawings $\Gamma_{a}$ and $\Gamma_{b}$ of any series-parallel graph $G$, the algorithm presented in Section 3.1 can be used to compute a pseudo-morph $M=\left\langle\Gamma_{a}, \ldots, \Gamma_{b}\right\rangle$. The idea is to augment both $\Gamma_{a}$ and $\Gamma_{b}$ to two drawings $\Gamma_{a}^{\prime}$ and $\Gamma_{b}^{\prime}$ of a biconnected series-parallel graph $G^{\prime}$, compute the morph $M^{\prime}=\left\langle\Gamma_{a}^{\prime}, \ldots, \Gamma_{b}^{\prime}\right\rangle$, and obtain $M$ by restricting $M^{\prime}$ to the vertices and edges of $G$.

This augmentation is performed on $G$ by repeatedly applying the following lemma.

Lemma 1. Let $v$ be a cut-vertex of a plane series-parallel graph $G$ with $n_{b}$ blocks. Let $e_{1}=(u, v)$ and $e_{2}=(w, v)$ be two consecutive edges in the circular order around $v$ such that $e_{1}$ belongs to block $b_{1}$ of $G$ and $e_{2}$ belongs to block $b_{2} \neq b_{1}$ of $G$. The graph $G^{*}$ obtained from $G$ by adding a vertex $z$ and edges $(u, z)$ and $(w, z)$ is a plane series-parallel graph with $n_{b}-1$ blocks.

Observe that, when augmenting $G$ to $G^{*}$, both $\Gamma_{a}$ and $\Gamma_{b}$ can be augmented to two planar straight-line drawings $\Gamma_{a}^{*}$ and $\Gamma_{b}^{*}$ of $G^{*}$ by placing vertex $z$ suitably close to $v$ and with direct visibility to vertices $u$ and $w$, as in the proof of Fáry's Theorem [9]. By repeatedly applying such an augmentation we obtain a biconnected series-parallel graph $G^{\prime}$ and its drawings $\Gamma_{a}^{\prime}$ and $\Gamma_{b}^{\prime}$, whose number of vertices and edges is linear in 
the size of $G$. Hence, the algorithm described in Section 3.1 can be applied to obtain a pseudo-morph $\left\langle\Gamma_{a}, \ldots, \Gamma_{b}\right\rangle$, thus proving Theorem 3. We will show in Section 5 how to obtain a morph starting from the pseudo-morph computed in this section.

\section{Morphing Plane Graph Drawings in $\mathrm{O}\left(\mathrm{n}^{2}\right)$ Steps}

In this section we prove the following theorem.

Theorem 4. Let $\Gamma_{s}$ and $\Gamma_{t}$ be two drawings of the same plane graph $G$. There exists a pseudo-morph $\left\langle\Gamma_{s}, \ldots, \Gamma_{t}\right\rangle$ with $O\left(n^{2}\right)$ steps transforming $\Gamma_{s}$ into $\Gamma_{t}$.

Preliminary Definitions. Let $\Gamma$ be a planar straight-line drawing of a plane graph $G$. A face $f$ of $G$ is empty in $\Gamma$ if it is delimited by a simple cycle. Consider a vertex $v$ of $G$ and let $v_{1}$ and $v_{2}$ be two of its neighbors. Vertices $v_{1}$ and $v_{2}$ are consecutive neighbors of $v$ if no edge appears between edges $\left(v, v_{1}\right)$ and $\left(v, v_{2}\right)$ in the circular order of the edges around $v$ in $\Gamma$. Let $v$ be a vertex with $\operatorname{deg}(v) \leq 5$ such that each face containing $v$ on its boundary is empty. We say that $v$ is contractible [1] if, for each two neighbors $u_{1}$ and $u_{2}$ of $v$, edge $\left(u_{1}, u_{2}\right)$ exists in $G$ if and only if $u_{1}$ and $u_{2}$ are consecutive neighbors of $v$. We say that $v$ is quasi-contractible if, for each two neighbors $u_{1}$ and $u_{2}$ of $v$, edge $\left(u_{1}, u_{2}\right)$ exists in $G$ only if $u_{1}$ and $u_{2}$ are consecutive neighbors of $v$. In other words, no edge exists between non-consecutive neighbors of a contractible or quasi-contractible vertex; also, each face incident to a contractible vertex $v$ is delimited by a 3-cycle, while a face incident to a quasi-contractible vertex might have more than three incident vertices. We have the following.

Lemma 2. Every planar graph contains a quasi-contractible vertex.

Further, given a neighbor $x$ of $v$, we say that $v$ is $x$-contractible onto $x$ in $\Gamma$ if: $(i) v$ is quasi-contractible, and (ii) the contraction of $v$ onto $x$ in $\Gamma$ results in a straight-line planar drawing $\Gamma^{\prime}$ of $G^{\prime}=G /(v, x)$.

The Algorithm. We describe the main steps of our algorithm to pseudo-morph a drawing $\Gamma_{s}$ of a plane graph $G$ into another drawing $\Gamma_{t}$ of $G$.

First, we consider a quasi-contractible vertex $v$ of $G$, that exists by Lemma 2 Second, we compute a pseudo-morph with $O(n)$ steps of $\Gamma_{s}$ into a drawing $\Gamma_{s}^{x}$ of $G$ and a pseudo-morph with $O(n)$ steps of $\Gamma_{t}$ into a drawing $\Gamma_{t}^{x}$ of $G$, such that $v$ is $x$ contractible onto the same neighbor $x$ both in $\Gamma_{s}^{x}$ and in $\Gamma_{t}^{x}$. We will describe later how to perform these pseudo-morphs. Third, we contract $v$ onto $x$ both in $\Gamma_{s}^{x}$ and in $\Gamma_{t}^{x}$, hence obtaining two drawings $\Gamma_{s}^{\prime}$ and $\Gamma_{t}^{\prime}$ of a graph $G^{\prime}=G /(v, x)$ with $n-1$ vertices. Fourth, we recursively compute a pseudo-morph transforming $\Gamma_{s}^{\prime}$ into $\Gamma_{t}^{\prime}$. This completes the description of the algorithm for constructing a pseudo-morphing transforming $\Gamma_{s}$ into $\Gamma_{t}$. Observe that the algorithm has $p(n) \in O\left(n^{2}\right)$ steps, thus proving Theorem 4. Namely, as it will be described later, $O(n)$ steps suffice to construct pseudo-morphings of $\Gamma_{s}$ and $\Gamma_{t}$ into drawings $\Gamma_{s}^{x}$ and $\Gamma_{t}^{x}$ of $G$, respectively, such that $v$ is $x$-contractible onto the same neighbor $x$ both in $\Gamma_{s}^{x}$ and in $\Gamma_{t}^{x}$. Further, two steps are sufficient to contract $v$ onto $x$ in both $\Gamma_{s}^{x}$ and $\Gamma_{t}^{x}$, obtaining drawings $\Gamma_{s}^{\prime}$ 


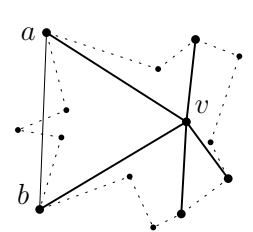

(a)

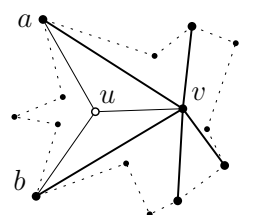

(b)

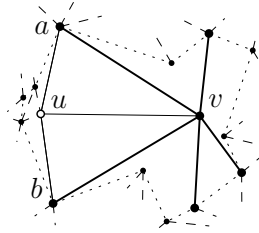

(c)

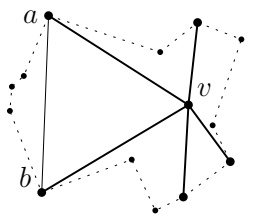

(d)

Fig. 4. Vertex $v$ and its neighbors. (a) Vertices $a$ and $b$ do not have direct visibility and the triangle $\langle a, b, v\rangle$ is not empty. (b) A vertex $u$ is added suitably close to $v$ and connected to $v, a$, and $b$. (c) The output of CONVEXIFIER on the quadrilateral $\langle a, b, v, u\rangle$. (d) Vertex $u$ and its incident edges can be removed in order to insert edge $(a, b)$.

and $\Gamma_{t}^{\prime}$, respectively. Finally, the recursion on $\Gamma_{s}^{\prime}$ and $\Gamma_{t}^{\prime}$ takes $p(n-1)$ steps. Thus, $p(n)=p(n-1)+O(n) \in O\left(n^{2}\right)$. We will show in Section 5 how to obtain a morph starting from the pseudo-morph computed in this section.

We remark that our approach is similar to the one proposed by Alamdari et al. [1]. In [1] $\Gamma_{s}$ and $\Gamma_{t}$ are augmented to drawings of the same maximal planar graph with $m \in$ $O\left(n^{2}\right)$ vertices, and a morph with $O\left(m^{2}\right)$ steps is constructed between two drawings of the same $m$-vertex maximal planar graph. This results in a morphing between $\Gamma_{s}$ and $\Gamma_{t}$ with $O\left(n^{4}\right)$ steps. Here, we also augment $\Gamma_{s}$ and $\Gamma_{t}$ to drawings of maximal planar graphs. However, we only require that the two maximal planar graphs coincide in the subgraph induced by the neighbors of $v$. Since this can be achieved by adding a constant number of vertices to $\Gamma_{s}$ and $\Gamma_{t}$, namely one for each of the at most five faces $v$ is incident to, our morphing algorithm has $O\left(n^{2}\right)$ steps.

Making $\boldsymbol{v} \boldsymbol{x}$-contractible. Let $v$ be a quasi-contractible vertex of $G$. We show an algorithm to construct a pseudo-morph with $O(n)$ steps transforming any straight-line planar drawing $\Gamma$ of $G$ into a straight-line planar drawing $\Gamma^{\prime}$ of $G$ such that $v$ is $x$ contractible onto any neighbor $x$. If $v$ has degree 1 , then it is contractible into its unique neighbor in $\Gamma$, and there is nothing to prove.

In order to transform $\Gamma$ into $\Gamma^{\prime}$, we use a support graph $S$ and its drawing $\Sigma$, initially set equal to $G$ and $\Gamma$, respectively. The goal is to augment $S$ and $\Sigma$ so that $v$ becomes a contractible vertex of $S$. In order to do this, we have to add to $S$ an edge between every two consecutive neighbors of $v$. However, the insertion of these edges might not be possible in $\Sigma$, as it might lead to a crossing or to enclose some vertex inside a cycle delimited by $v$ and by two consecutive neighbors of $v$ (see Fig. 4(a)).

Let $a$ and $b$ be two consecutive neighbors of $v$. If the closed triangle $\langle a, b, v\rangle$ does not contain any vertex other than $a, b$, and $v$, then add edge $(a, b)$ to $S$ and to $\Sigma$ as a straight-line segment. Otherwise, proceed as follows.

Let $\Sigma_{u}$ be the drawing of a plane graph $S_{u}$ obtained by adding a vertex $u$ and the edges $(u, v),(u, a)$, and $(u, b)$ to $\Sigma$ and to $S$, in such a way that the resulting drawing is straight-line planar and each face containing $u$ on its boundary is empty. As in the proof of Fáry's Theorem [9], a position for $u$ with such properties can be found in $\Sigma$, suitably close to $v$. See Fig. 4(b). Augment $\Sigma_{u}$ to the drawing $\Theta$ of a maximal plane graph $T$ 
by first adding three vertices $p, q$, and $r$ to $\Sigma_{u}$, so that triangle $\langle p, q, r\rangle$ encloses the rest of the drawing, and then adding dummy edges [8]. If edge $(a, b)$ has been added in this augmentation (this can happen if $a$ and $b$ share a face not having $v$ on its boundary), subdivide $(a, b)$ in $\Theta$ (namely, replace $(a, b)$ with edges $(a, w)$ and $(w, b)$, placing $w$ along the straight-line segment connecting $a$ and $b$ ) and triangulate the two faces vertex $w$ is incident to. Next, apply the algorithm described in [1], that we call CONVEXIFIER, to construct a morph of $\Theta$ into a drawing $\Theta^{\prime}$ of $T$ in which polygon $\langle a, v, b, u\rangle$ is convex. The input of algorithm CONVEXIFIER consists of a planar straight-line drawing $\Gamma^{*}$ of a plane graph $G^{*}$ and of a set of at most five vertices of $G^{*}$ inducing a biconnected outerplane graph not containing any other vertex in its interior in $\Gamma^{*}$. The output of algorithm CONVEXIFIER is a sequence of $O(n)$ linear morphing steps transforming $\Gamma^{*}$ into a drawing of $G^{*}$ in which the at most five input vertices bound a convex polygon. Since, by construction, vertices $a, v, b, u$ satisfy all such requirements, we can apply algorithm CONVEXIFIER to $\Theta$ and to $a, v, b, u$, hence obtaining a morph with $O(n)$ steps transforming $\Theta$ into the desired drawing $\Theta^{\prime}$ (see Fig. 4(c)). Let $\Sigma_{u}^{\prime}$ be the drawing of $S_{u}$ obtained by restricting $\Theta^{\prime}$ to vertices and edges of $S_{u}$. Since $\langle a, v, b, u\rangle$ is a convex polygon containing no vertex of $S_{u}$ in its interior, edge $(u, v)$ can be removed from $\Sigma_{u}^{\prime}$ and an edge $(a, b)$ can be introduced in $\Sigma_{u}^{\prime}$, so that the resulting drawing $\Sigma^{\prime}$ is planar and cycle $(a, b, v)$ does not contain any vertex in its interior (see Fig. 4(d)).

Once edge $(a, b)$ has been added to $S$ (either in $\Sigma$ or after the described procedure transforming $\Sigma$ into $\Sigma^{\prime}$ ), if $\operatorname{deg}(v)=2$ then $v$ is both $a$-contractible and $b$-contractible. Otherwise, consider a new pair of consecutive vertices of $v$ not creating an empty triangular face with $v$, if any, and apply the same operations described before.

Once every pair of consecutive vertices has been handled, vertex $v$ is contractible in $S$. Let $\Sigma_{v}$ be the current drawing of $S$. Augment $\Sigma_{v}$ to the drawing $\Theta_{v}$ of a triangulation $T_{v}$ (by adding three vertices and a set of edges), contract $v$ onto a neighbor $w$ such that $v$ is $w$-contractible (one of such neighbors always exists, given that $v$ is contractible), and apply CONVEXIFIER to the resulting drawing $\Theta_{v}^{\prime}$ and to the neighbors of $v$ to construct a morphing $\Theta_{v}^{\prime}$ to a drawing $\Sigma_{v}^{\prime}$ in which the polygon defined by such vertices is convex. Drawing $\Gamma^{\prime}$ of $G$ in which $v$ is $x$-contractible for any neighbor $x$ of $v$ is obtained by restricting $\Sigma_{v}^{\prime}$ to the vertices and the edges of $G$. We can now contract $v$ onto $x$ in $\Gamma^{\prime}$ and recur on the obtained graph (with $n-1$ vertices) and drawing.

It remains to observe that, given a quasi-contractible vertex $v$, the procedure to construct a pseudo-morph of $\Gamma$ into $\Gamma^{\prime}$ consists of at most $\operatorname{deg}(v)+1$ executions of CONVEXIFIER, each requiring a linear number of steps [1]. As $\operatorname{deg}(v) \leq 5$, the procedure to pseudo-morph $\Gamma$ into $\Gamma^{\prime}$ has $O(n)$ steps. This concludes the proof of Theorem 4 .

\section{Transforming a Pseudo-Morph into a Morph}

In this section we show how to obtain an actual morph $M$ from a given pseudo-morph $\mathcal{M}$, by describing how to compute the placement and the motion of any vertex $v$ that has been contracted during $\mathcal{M}$. By applying this procedure to Theorems 3 and 4 , we obtain a proof of Theorems 1 and 2 . 
Let $\Gamma$ be a drawing of a graph $G$ and let $\mathcal{M}=\left\langle\Gamma, \ldots, \Gamma^{*}\right\rangle$ be a pseudo-morph that consists of the contraction of a vertex $v$ of $G$ onto one of its neighbors $x$, followed by a pseudo-morph $\mathcal{M}^{\prime}$ of the graph $G^{\prime}=G /(v, x)$, and then of the uncontraction of $v$.

The idea of how to compute $M$ from $\mathcal{M}$ is the same as in [1]: Namely, morph $M$ is obtained by $(i)$ recursively converting $\mathcal{M}^{\prime}$ into a morph $M^{\prime} ;(i i)$ modifying $M^{\prime}$ to a morph $M_{v}^{\prime}$ obtained by adding vertex $v$ (and its incident edges) to each drawing of $M^{\prime}$, in a suitable position; (iii) replacing the contraction of $v$ onto $x$, performed in $\mathcal{M}$, with a linear morph that moves $v$ from its initial position in $\Gamma$ to its position in the first drawing of $M_{v}^{\prime}$; and $(i v)$ replacing the uncontraction of $v$, performed in $\mathcal{M}$, with a linear morph that moves $v$ from its position in the last drawing of $M_{v}^{\prime}$ to its final position in $\Gamma^{*}$. Note that, in order to guarantee the planarity of $M$ when adding $v$ to any drawing of $M^{\prime}$ in order to obtain $M_{v}^{\prime}$, vertex $v$ must lie inside its kernel. Since vertex $x$ lies in the kernel of $v$ (as $x$ is adjacent to all the neighbors of $v$ in $G^{\prime}$ ), we achieve this property by placing $v$ suitably close to $x$, as follows.

At any time instant $t$ during $M^{\prime}$, there exists an $\epsilon_{t}>0$ such that the disk $D$ centered at $x$ with radius $\epsilon_{t}$ does not contain any vertex other than $x$. Let $\epsilon$ be the minimum among the $\epsilon_{t}$ during $M^{\prime}$. We place vertex $v$ at a suitable point of a sector $S$ of $D$ according to the following cases. Case (a); $\boldsymbol{v}$ has degree 1 in $\boldsymbol{G}$. Sector $S$ is defined as the intersection of $D$ with the face containing $v$ in $G$. See Fig. 5(a). Case (b); $v$ has degree 2 in $G$. Sector $S$ is defined as the intersection of $D$ with the face containing $v$ in $G$ and with the halfplane defined by the straight-line passing through $x$ and $r$, and containing $v$ in $\Gamma$. See Fig. 5(b). Otherwise, $\operatorname{deg}(v) \geq 3$ in $G^{\prime}$. Let $(r, v)$ and $(l, v)$ be the two edges such that $(r, v),(x, v)$, and $(l, v)$ are clockwise consecutive around $v$ in $G$. Observe that edges $(r, x)$ and $(l, x)$ exist in $G^{\prime}$. Assume that $x, r$, and $l$ are not collinear in any drawing of $M^{\prime}$, as otherwise we can slightly perturb such a drawing without compromising the planarity of $M^{\prime}$. Let $\alpha_{i}$ be the angle $\widehat{l x r}$ in any intermediate drawing of $M^{\prime}$. Case (c); $\boldsymbol{\alpha}_{\boldsymbol{i}}<\pi$. Sector $S$ is defined as the intersection of $D$ with the wedge delimited by edges $(x, r)$ and $(x, l)$. See Fig. 5(c). Case (d); $\boldsymbol{\alpha}_{\boldsymbol{i}}>\pi$. Sector $S$ is defined as the intersection of $D$ with the wedge delimited by the elongations of $(x, r)$ and $(x, l)$ emanating from $x$. See Fig. 5(d). By exploiting the techniques shown in [1], the motion of $v$ can be computed according to the evolution of $S$ over $M^{\prime}$, thus obtaining a planar morph $M_{v}^{\prime}$.

Observe that, in the algorithm described in Section 4, the vertex $x$ onto which $v$ has been contracted might be not adjacent to $v$ in $G$. However, since a contraction has been performed, $x$ is adjacent to $v$ in one of the graphs obtained when augmenting $G$ during the algorithm. Hence, a morph of $G$ can be obtained by applying the above procedure to the pseudo-morph computed on this augmented graph and by restricting it to the vertices and edges of $G$.

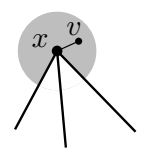

(a)

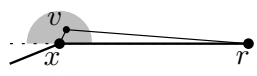

(b)

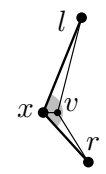

(c)

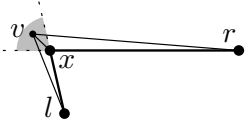

(d)

Fig. 5. Sector $S$ (in grey) when:(a) $\operatorname{deg}(v)=1$, (b) $\operatorname{deg}(v)=2$, and (c) (d) $\operatorname{deg}(v) \geq 3$. 


\section{Conclusions and Open Problems}

In this paper we studied the problem of designing efficient algorithms for morphing two planar straight-line drawings of the same graph. We proved that any two planar straightline drawings of a series-parallel graph can be morphed with $O(n)$ linear morphing steps, and that a planar morph with $O\left(n^{2}\right)$ linear morphing steps exists between any two planar straight-line drawings of any planar graph.

It is a natural open question whether the bounds we presented are optimal or not. We suspect that planar straight-line drawings exist requiring a linear number of steps to be morphed one into the other. However, no super-constant lower bound for the number of morphing steps required to morph planar straight-line drawings is known. It would be interesting to understand whether our techniques can be extended to compute morphs between any two drawings of a partial planar 3-tree with a linear number of steps. We recall that, as observed in [1], a linear number of morphing steps suffices to morph any two drawings of a maximal planar 3-tree.

\section{References}

1. Alamdari, S., Angelini, P., Chan, T.M., Di Battista, G., Frati, F., Lubiw, A., Patrignani, M., Roselli, V., Singla, S., Wilkinson, B.T.: Morphing planar graph drawings with a polynomial number of steps. In: SODA 2013, pp. 1656-1667 (2013)

2. Angelini, P., Cortese, P.F., Di Battista, G., Patrignani, M.: Topological morphing of planar graphs. In: Tollis, I.G., Patrignani, M. (eds.) GD 2008. LNCS, vol. 5417, pp. 145-156. Springer, Heidelberg (2009)

3. Angelini, P., Didimo, W., Kobourov, S., Mchedlidze, T., Roselli, V., Symvonis, A., Wismath, S.: Monotone drawings of graphs with fixed embedding. Algorithmica, 1-25 (2013)

4. Angelini, P., Frati, F., Patrignani, M., Roselli, V.: Morphing planar graph drawings efficiently. CoRR cs.CG (2013), http: / / arxiv.org/abs /1308.4291

5. Angelini, P., Colasante, E., Di Battista, G., Frati, F., Patrignani, M.: Monotone drawings of graphs. J. of Graph Algorithms and Appl. 16(1), 5-35 (2012); In: Brandes, U., Cornelsen, S. (eds.) GD 2010. LNCS, vol. 6502, pp. 13-24. Springer, Heidelberg (2011)

6. Biedl, T.C., Lubiw, A., Spriggs, M.J.: Morphing planar graphs while preserving edge directions. In: Healy, P., Nikolov, N.S. (eds.) GD 2005. LNCS, vol. 3843, pp. 13-24. Springer, Heidelberg (2006)

7. Cairns, S.S.: Deformations of plane rectilinear complexes. American Math. Monthly 51, 247-252 (1944)

8. Chazelle, B.: Triangulating a simple polygon in linear time. Discrete \& Computational Geometry 6(5), 485-524 (1991)

9. Fáry, I.: On straight line representation of planar graphs. Acta Univ. Szeged. Sect. Sci. Math. 11, 229-233 (1948)

10. Grunbaum, B., Shephard, G.: The geometry of planar graphs. Cambridge University Press (1981), http://dx.doi.org/10.1017/CB09780511662157.008

11. Lubiw, A., Petrick, M.: Morphing planar graph drawings with bent edges. Electronic Notes in Discrete Mathematics 31, 45-48 (2008)

12. Lubiw, A., Petrick, M., Spriggs, M.: Morphing orthogonal planar graph drawings. In: SODA 2006, pp. 222-230. ACM (2006)

13. Thomassen, C.: Deformations of plane graphs. J. Comb. Th., Series B 34, 244-257 (1983) 